\title{
Supplment to Interim Report on Task 1.3: Equilibrium Phase Diagram to Lawrence Livermore National Laboratory for Contract B345772
}

M. W. A. Stewart, E. R. Vance, and R. A. Day

\section{April 18, 1999}


This document was prepared as an account of work sponsored by an agency of the United States Government. Neither the United States Government nor the University of California nor any of their employees, makes any warranty, express or implied, or assumes any legal liability or responsibility for the accuracy, completeness, or usefulness of any information, apparatus, product, or process disclosed, or represents that its use would not infringe privately owned rights. Reference herein to any specific commercial product, process, or service by trade name, trademark, manufacturer, or otherwise, does not necessarily constitute or imply its endorsement, recommendation, or favoring by the United States Government or the University of California. The views and opinions of authors expressed herein do not necessarily state or reflect those of the United States Government or the University of California, and shall not be used for advertising or product endorsement purposes.

This work was performed under the auspices of the U.S. Department of Energy by University of California, Lawrence Livermore National Laboratory under Contract W-7405-Eng-48. 
Supplement to

Interim Report on Task 1.3: Equilibrium Phase Diagram

\section{to Lawrence Livermore National Laboratory for Contract B345772 -}

M W A Stewart, E R Vance and R A Day

18 April 1999

$\mathrm{R} 99 \mathrm{m023}$ 


\section{Supplement to}

\section{Interim Report on Task 1.3: Equilibrium Phase Diagram}

DATE ISSUED

18 April 1999

ISSUED TO

Lawrence Livermore National Laboratory

REPORT NUMBER

$\mathrm{R} 99 \mathrm{~m} 023$

AUTHORS

Stewart, Vance, Day,
JOB NUMBER

$713 m$

APPROVED BY

E R Vance

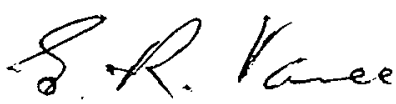

Australian Nuclear Science and Technology Organisation Postal Address: Private Mail Bag 1, Menai, NSW 2234, Australia Materials Division: Telephone +61297173265 - Facsimile +61295437179 


\title{
Task 1: Immobilised Form Development
}

\author{
Supplementary Report
}

Task 1.3 Equilibrium Phase Diagram 


\section{Summary}

This report contains a summary of the results generated so far on the $\mathrm{CaHfTi}_{2} \mathrm{O}_{7}-$ $\mathrm{Gd}_{2} \mathrm{Ti}_{2} \mathrm{O}_{7}$ System.

\section{Experimental}

Samples were made using alkoxides and nitrates (Table 1), via the alkoxide-route discussed in a previous report ${ }^{1}$. Compositions made are given in Table 2.

Analysis was carried out by $\mathrm{x}$-ray diffraction (XRD) and scanning electron microscopy SEM), with energy dispersive $\mathrm{x}$-ray spectrometry (EDS) used for quantitative analysis. Details have been given previously ${ }^{1}$.

Samples were sintered on Pt foil in air at $1500^{\circ} \mathrm{C}$ for 100 hours.

Table 1: Raw materials used in Task 1.3

\begin{tabular}{|l|l|}
\hline Element & $\begin{array}{l}\text { Raw Materials for Alkoxide-route Batches (raw material, source, catalogue } \\
\text { number) }\end{array}$ \\
\hline $\mathrm{Ca}$ & $99 \% \mathrm{Ca}\left(\mathrm{NO}_{3}\right)_{2} \cdot 4 \mathrm{H}_{2} \mathrm{O}$, Aldrich Chem. Co., 23712-4 \\
$\mathrm{Gd}$ & $99.9 \% \mathrm{Gd}\left(\mathrm{NO}_{3}\right)_{3} \cdot 6 \mathrm{H}_{2} \mathrm{O}$, Aldrich Chem., 21159-1 \\
$\mathrm{Hf}$ & $99.99 \%$ Hafnium n-butoxide, Gelest Inc. \\
$\mathrm{Ti}$ & Titanium isopropoxide, HÜLS Troisdorf Gmb., 405514 \\
\hline
\end{tabular}

\section{Results and Discussion}

The phases detected in the samples are given in Table 2. The EDS results are given in Tables 3 to 5. Typical microstructures are given in Figures 1 to 4 . The $x$-ray diffraction paterns for the sample are given in Appendix A.

There are well defined transition zones with $4 \mathrm{M}$ zirconolite appearing in the $\mathrm{Ca}_{0.45} \mathrm{Gd}_{1.1} \mathrm{Hf}_{0.45} \mathrm{Ti}_{2} \mathrm{O}_{7}$ sample and $2 \mathrm{M}$ zirconolite appearing in the $\mathrm{Ca}_{0.8} \mathrm{Gd}_{0.4} \mathrm{Hf}_{0.8} \mathrm{Ti}_{2} \mathrm{O}_{7}$ sample. For the zirconolite composition $\left(\mathrm{Ca}_{z} \mathrm{Gd}_{\mathrm{y}} \mathrm{Hf}_{\mathrm{x}} \mathrm{Ti}_{2} \mathrm{O}_{7}\right)$, the $4 \mathrm{M}$ polytype covers the approximate range $0.5<\mathrm{x}<0.66$. The $2 \mathrm{M}$ polytype occurs for $\mathrm{x}>0.8$.

\footnotetext{
${ }^{1}$ M.W.A. Stewart, E.R. Vance, R.A. Day and A. Brownscombe, "Interim Report on Task 1.2: Near equilibrium Requirements, ANSTO Report No. R99m012, 5 April, 1999.
} 
The pyrochlore composition is close to the design composition. The maximum amount of Hf measured in the pyrochlore in the samples was 0.42 formula units.

Excess rutile samples mws 980400 and 401 show some zoning around the edges of the grains (tables 3 and 4). This was not observed in samples without excess rutile.

The rutile incorporates some hafnium (Table 5) and hence, in samples made with excess titania, the pyrochlore and zirconolite are deficient in hafnia, relative to the designed composition (Tables 3 and 4). The rutile composition is relatively constant $\left(\sim \mathrm{Hf}_{0.1} \mathrm{Ti}_{0.9} \mathrm{O}_{2}\right)$ except near the zirconolite end of the test spectrum, where the rutile has slightly more Hf (Table 5). The rutile in samples made with excess titania has slightly less Hf.

\section{Conclusion}

The pyrochlore-zirconolite boundary is observed at approximately -

$0.6\left(\mathrm{Gd}_{0.6} \mathrm{Ti}_{2} \mathrm{O}_{7}\right)+0.4\left(\mathrm{CaHfTi}_{2} \mathrm{O}_{7}\right)$. 
Table 2: Compositions tested and phases detected in SEM

\begin{tabular}{|c|c|c|c|}
\hline Sample No. & Pyrochlore & $\begin{array}{l}\text { Excess } \mathrm{TiO}_{2} \text { or } \\
\mathrm{HfO}_{2} \\
\text { (wt. \%) }\end{array}$ & Phases detected \\
\hline mws 980399 & $\mathrm{Ca}_{0.3} \mathrm{Gd}_{1.4} \mathrm{Hf}_{0.3} \mathrm{Ti}_{2} \mathrm{O}_{7}$ & $18.2 \% \mathrm{TiO}_{2}$ & Pyrochlore + rutile \\
\hline mws 980420 & $\mathrm{Ca}_{0.3} \mathrm{Gd}_{1.4} \mathrm{Hf}_{0.3} \mathrm{Ti}_{2} \mathrm{O}_{7}$ & $2 \% \mathrm{HfO}_{2}$ & Pyrochlore + rutile \\
\hline mws980403 & $\mathrm{Ca}_{0.35} \mathrm{Gr}_{0.3} \mathrm{Hf}_{0.35} \mathrm{Ti}_{2} \mathrm{O}_{7}$ & & Pyrochlore + rutile \\
\hline mws 980400 & $\mathrm{Ca}_{0.4} \mathrm{Gd}_{1.2} \mathrm{Hf}_{0.4} \mathrm{Ti}_{2} \mathrm{O}_{7}$ & $18.6 \% \mathrm{TiO}_{2}$ & Pyrochlore + rutile \\
\hline mws980421 & $\mathrm{Ca}_{0.4} \mathrm{Gd}_{1.2} \mathrm{Hf}_{0.4} \mathrm{Ti}_{2} \mathrm{O}_{7}$ & $2 \% \mathrm{HfO}_{2}$ & Pyrochlore + rutile \\
\hline mws 980402 & $\mathrm{Ca}_{0.45} \mathrm{Gd}_{1.1} \mathrm{Hf}_{0.45} \mathrm{Ti}_{2} \mathrm{O}_{7}$ & & $\begin{array}{l}\text { Pyrochlore }+4 \mathrm{M} \\
\text { zirconolite + rutile }\end{array}$ \\
\hline mws980401 & $\mathrm{Ca}_{0.5} \mathrm{Gd}_{1.0} \mathrm{Hf}_{0.5} \mathrm{Ti}_{2} \mathrm{O}_{7}$ & $18.9 \% \mathrm{TiO}_{2}$ & $\begin{array}{l}\text { Pyrochlore }+4 \mathrm{M} \\
\text { zirconolite + rutile }\end{array}$ \\
\hline mws 980422 & $\mathrm{Ca}_{0.5} \mathrm{Gd}_{1.0} \mathrm{Hf}_{0.5} \mathrm{Ti}_{2} \mathrm{O}_{7}$ & $2 \% \mathrm{HfO}_{2}$ & $\begin{array}{l}\text { Pyrochlore }+4 \mathrm{M} \\
\text { zirconolite + rutile }\end{array}$ \\
\hline mws 980423 & $\mathrm{Ca}_{0.6} \mathrm{Gd}_{0.8} \mathrm{Hf}_{0.6} \mathrm{Ti}_{2} \mathrm{O}_{7}$ & & $4 \mathrm{M}$ zirconolite + rutile \\
\hline mws 980424 & $\mathrm{Ca}_{0.7} \mathrm{Gd}_{0.6} \mathrm{Hf}_{0.7} \mathrm{Ti}_{2} \mathrm{O}_{7}$ & & $4 \mathrm{M}$ zirconolite + rutile \\
\hline mws 980425 & $\mathrm{Ca}_{0.8} \mathrm{Gd}_{0.4} \mathrm{Hf}_{0.8} \mathrm{Ti}_{2} \mathrm{O}_{7}$ & & $\begin{array}{l}2 \mathrm{M} \text { zirconolite }+4 \mathrm{M} \\
\text { zirconolite }+ \text { rutile }\end{array}$ \\
\hline
\end{tabular}


Table 3: Composition of pyrochlore phase in the samples

\begin{tabular}{|c|c|c|c|}
\hline $\begin{array}{l}\text { Sample } \\
\text { No. }\end{array}$ & Pyrochlore & $\begin{array}{l}\text { Excess } \mathrm{TiO}_{2} \text { or } \\
\mathrm{HfO}_{2} \\
\text { (wt. \%) }\end{array}$ & Pyrochlore composition \\
\hline mws980399 & $\mathrm{Ca}_{0.3} \mathrm{Gd}_{1.4} \mathrm{Hf}_{0.3} \mathrm{Ti}_{2} \mathrm{O}_{7}$ & $18.2 \% \mathrm{TiO}_{2}$ & $\mathrm{Ca}_{0.29} \mathrm{Gd}_{1.42} \mathrm{Hf}_{0.22} \mathrm{Ti}_{2.07} \mathrm{O}_{7}$ \\
\hline mws 980420 & $\mathrm{Ca}_{0.3} \mathrm{Gd}_{1.4} \mathrm{Hf}_{0.3} \mathrm{Ti}_{2} \mathrm{O}_{7}$ & $2 \% \mathrm{HfO}_{2}$ & $\mathrm{Ca}_{0.28} \mathrm{Gd}_{1.43} \mathrm{Hf}_{0.32} \mathrm{Ti}_{1.96} \mathrm{O}_{7}$ \\
\hline mws980403 & $\mathrm{Ca}_{0.35} \mathrm{Gd}_{1.3} \mathrm{Hf}_{0.35} \mathrm{Ti}_{2} \mathrm{O}_{7}$ & & $\mathrm{Ca}_{0.35} \mathrm{Gd}_{1.34} \mathrm{Hf}_{0.35} \mathrm{Ti}_{2.00} \mathrm{O}_{7}$ \\
\hline mws 980400 & $\mathrm{Ca}_{0.4} \mathrm{Gd}_{1.2} \mathrm{Hf}_{0.4} \mathrm{Ti}_{2} \mathrm{O}_{7}$ & $18.6 \% \mathrm{TiO}_{2}$ & $\begin{array}{l}\mathrm{Ca}_{0.40} \mathrm{Gd}_{1.29} \mathrm{Hf}_{0.29} \mathrm{Ti}_{2.04} \mathrm{O}_{7} \\
\text { darker zone around edge of } \\
\text { pyrochlore grains - } \\
\mathrm{Ca}_{0.44} \mathrm{Gd}_{1.20} \mathrm{Hf}_{0.27} \mathrm{Ti}_{2.11} \mathrm{O}_{7}\end{array}$ \\
\hline mws980421 & $\mathrm{Ca}_{0.4} \mathrm{Gd}_{1.2} \mathrm{Hf}_{0.4} \mathrm{Ti}_{2} \mathrm{O}_{7}$ & $2 \% \mathrm{HfO}_{2}$ & $\mathrm{Ca}_{0.40} \mathrm{Gd}_{1.26} \mathrm{Hf}_{0.41} \mathrm{Ti}_{1.95} \mathrm{O}_{7}$ \\
\hline mws 980402 & $\mathrm{Ca}_{0.45} \mathrm{Gd}_{1.1} \mathrm{Hf}_{0.45} \mathrm{Ti}_{2} \mathrm{O}_{7}$ & & $\mathrm{Ca}_{0.44} \mathrm{Gd}_{1.19} \mathrm{Hf}_{0.40} \mathrm{Ti}_{1.98} \mathrm{O}_{7}$ \\
\hline mws 980401 & $\mathrm{Ca}_{0.5} \mathrm{Gd}_{1.0} \mathrm{Hf}_{0.5} \mathrm{Ti}_{2} \mathrm{O}_{7}$ & $18.9 \% \mathrm{TiO}_{2}$ & $\mathrm{Ca}_{0.46} \mathrm{Gd}_{1.14} \mathrm{Hf}_{0.35} \mathrm{Ti}_{2.06} \mathrm{O}_{7}$ \\
\hline mws 980422 & $\mathrm{Ca}_{0.5} \mathrm{Gd}_{1.0} \mathrm{Hf}_{0.5} \mathrm{Ti}_{2} \mathrm{O}_{7}$ & $2 \% \mathrm{HfO}_{2}$ & $\mathrm{Ca}_{0.42} \mathrm{Gd}_{1.19} \mathrm{Hf}_{0.42} \mathrm{Ti}_{1.97} \mathrm{O}_{7}$ \\
\hline mws 980423 & $\mathrm{Ca}_{0.6} \mathrm{Gd}_{0.8} \mathrm{Hf}_{0.6} \mathrm{Ti}_{2} \mathrm{O}_{7}$ & & NIL \\
\hline mws 980424 & $\mathrm{Ca}_{0.7} \mathrm{Gd}_{0.6} \mathrm{Hf}_{0.7} \mathrm{Ti}_{2} \mathrm{O}_{7}$ & & NIL \\
\hline mws 980425 & $\mathrm{Ca}_{0.8} \mathrm{Gd}_{0.4} \mathrm{Hf}_{0.8} \mathrm{Ti}_{2} \mathrm{O}_{7}$ & & NIL \\
\hline
\end{tabular}


Table 4: Composition of zirconolite phases in the samples

\begin{tabular}{|c|c|c|c|}
\hline $\begin{array}{l}\text { Sample } \\
\text { No. }\end{array}$ & Pyrochlore & $\begin{array}{l}\text { Excess } \mathrm{TiO}_{2} \text { or } \\
\mathrm{HfO}_{2} \\
\text { (wt. \%) }\end{array}$ & $\begin{array}{l}\text { Zirconolite composition } \\
\text { Polytype }\end{array}$ \\
\hline mws 980399 & $\mathrm{Ca}_{0.3} \mathrm{Gd}_{1.4} \mathrm{Hf}_{0.3} \mathrm{Ti}_{2} \mathrm{O}_{7}$ & $18.2 \% \mathrm{TiO}_{2}$ & NIL \\
\hline mws 980420 & $\mathrm{Ca}_{0.3} \mathrm{Gd}_{1.4} \mathrm{Hf}_{0.3} \mathrm{Ti}_{2} \mathrm{O}_{7}$ & $2 \% \mathrm{HfO}_{2}$ & NIL \\
\hline mws980403 & $\mathrm{Ca}_{0.35} \mathrm{Gd}_{1.3} \mathrm{Hf}_{0.35} \mathrm{Ti}_{2} \mathrm{O}_{7}$ & & NIL \\
\hline mws 980400 & $\mathrm{Ca}_{0.4} \mathrm{Gd}_{1.2} \mathrm{Hf}_{0.4} \mathrm{Ti}_{2} \mathrm{O}_{7}$ & $18.6 \% \mathrm{TiO}_{2}$ & $\mathrm{NIL}$ \\
\hline mws 980421 & $\mathrm{Ca}_{0.4} \mathrm{Gd}_{1.2} \mathrm{Hf}_{0.4} \mathrm{Ti}_{2} \mathrm{O}_{7}$ & $2 \% \mathrm{HfO}_{2}$ & NL \\
\hline mws 980402 & $\mathrm{Ca}_{0.45} \mathrm{Gd}_{1.1} \mathrm{Hf}_{0.45} \mathrm{Ti}_{2} \mathrm{O}_{7}$ & & $4 \mathrm{M}-\mathrm{Ca}_{0.54} \mathrm{Gd}_{0.86} \mathrm{Hf}_{0.57} \mathrm{Ti}_{1.98} \mathrm{O}_{7}$ \\
\hline mws980401 & $\mathrm{Ca}_{0.5} \mathrm{Gd}_{1.0} \mathrm{Hf}_{0.5} \mathrm{Ti}_{2} \mathrm{O}_{7}$ & $18.9 \% \mathrm{TiO}_{2}$ & $\begin{array}{l}4 \mathrm{M}-\mathrm{Ca}_{0.58} \mathrm{Gd}_{0.79} \mathrm{Hf}_{0.49} \mathrm{Ti}_{2.13} \mathrm{O}_{7} \\
\text { Darker overgrowth } 4 \mathrm{M}- \\
\mathrm{Ca}_{0.58} \mathrm{Gd}_{0.87} \mathrm{Hf}_{0.34} \mathrm{Ti}_{2.13} \mathrm{O}_{7}\end{array}$ \\
\hline mws 980422 & $\mathrm{Ca}_{0.5} \mathrm{Gd}_{1.0} \mathrm{Hf}_{0.5} \mathrm{Ti}_{2} \mathrm{O}_{7}$ & $2 \% \mathrm{HfO}_{2}$ & $4 \mathrm{M}-\mathrm{Ca}_{0.53} \mathrm{Gd}_{0.86} \mathrm{Hf}_{0.59} \mathrm{Ti}_{2.00} \mathrm{O}_{7}$ \\
\hline mws 980423 & $\mathrm{Ca}_{0.6} \mathrm{Gd}_{0.8} \mathrm{Hf}_{0.6} \mathrm{Ti}_{2} \mathrm{O}_{7}$ & & $4 \mathrm{M}-\mathrm{Ca}_{0.57} \mathrm{Gd}_{0.80} \mathrm{Hf}_{0.56} \mathrm{Ti}_{2.05} \mathrm{O}_{7}$ \\
\hline mws980424 & $\mathrm{Ca}_{0.7} \mathrm{Gd}_{0.6} \mathrm{Hf}_{0.7} \mathrm{Ti}_{2} \mathrm{O}_{7}$ & & $4 \mathrm{M}-\mathrm{Ca}_{0.68} \mathrm{Gd}_{0.63} \mathrm{Hf}_{0.66} \mathrm{Ti}_{2.02} \mathrm{O}_{7}$ \\
\hline mws 980425 & $\mathrm{Ca}_{0.8} \mathrm{Gd}_{0.4} \mathrm{Hf}_{0.8} \mathrm{Ti}_{2} \mathrm{O}_{7}$ & & $\begin{array}{l}4 \mathrm{M}-\mathrm{Ca}_{0.72} \mathrm{Gd}_{0.56} \mathrm{Hf}_{0.66} \mathrm{Ti}_{2.05} \mathrm{O}_{7} \\
2 \mathrm{M}-\mathrm{Ca}_{0.80} \mathrm{Gd}_{0.29} \mathrm{Hf}_{0.83} \mathrm{Ti}_{2.05} \mathrm{O}_{7}\end{array}$ \\
\hline
\end{tabular}


Table 5: Composition of rutile in the samples

\begin{tabular}{|c|c|c|c|}
\hline $\begin{array}{l}\text { Sample } \\
\text { No. }\end{array}$ & Pyrochlore & $\begin{array}{l}\text { Excess } \mathrm{TiO}_{2} \text { or } \\
\mathrm{HfO}_{2} \\
\text { (wt. \%) }\end{array}$ & Rutile composition \\
\hline mws980399 & $\mathrm{Ca}_{0.3} \mathrm{Gd}_{1.4} \mathrm{Hf}_{0.3} \mathrm{Ti}_{2} \mathrm{O}_{7}$ & $18.2 \% \mathrm{TiO}_{2}$ & $\mathrm{Hf}_{0.06} \mathrm{Ti}_{0.94} \mathrm{O}_{2}$ \\
\hline mws 980420 & $\mathrm{Ca}_{0.3} \mathrm{Gd}_{1.4} \mathrm{Hf}_{0.3} \mathrm{Ti}_{2} \mathrm{O}_{7}$ & $2 \% \mathrm{HfO}_{2}$ & $\mathrm{Hf}_{0.10} \mathrm{Ti}_{0.90} \mathrm{O}_{2}$ \\
\hline mws 980403 & $\mathrm{Ca}_{0.35} \mathrm{Gd}_{1.3} \mathrm{Hf}_{0.35} \mathrm{Ti}_{2} \mathrm{O}_{7}$ & & $\mathrm{Hf}_{0.09} \mathrm{Ti}_{0.91} \mathrm{O}_{2}$ \\
\hline mws 980400 & $\mathrm{Ca}_{0.4} \mathrm{Gd}_{1.2} \mathrm{Hf}_{0.4} \mathrm{Ti}_{2} \mathrm{O}_{7}$ & $18.6 \% \mathrm{TiO}_{2}$ & $\mathrm{Hf}_{0.07} \mathrm{Ti}_{0.93} \mathrm{O}_{2}$ \\
\hline mws980421 & $\mathrm{Ca}_{0.4} \mathrm{Gd}_{1.2} \mathrm{Hf}_{0.4} \mathrm{Ti}_{2} \mathrm{O}_{7}$ & $2 \% \mathrm{HfO}_{2}$ & $\mathrm{Hf}_{0.12} \mathrm{Ti}_{0.88} \mathrm{O}_{2}$ \\
\hline mws980402 & $\mathrm{Ca}_{0.45} \mathrm{Gd}_{1.1} \mathrm{Hf}_{0.45} \mathrm{Ti}_{2} \mathrm{O}_{7}$ & & $\mathrm{Hf}_{0.11} \mathrm{Ti}_{0.89} \mathrm{O}_{2}$ \\
\hline mws 980401 & $\mathrm{Ca}_{0.5} \mathrm{Gd}_{1.0} \mathrm{Hf}_{0.5} \mathrm{Ti}_{2} \mathrm{O}_{7}$ & $18.9 \% \mathrm{TiO}_{2}$ & $\mathrm{Hf}_{0.08} \mathrm{Ti}_{0.92} \mathrm{O}_{2}$ \\
\hline mws 980422 & $\mathrm{Ca}_{0.5} \mathrm{Gd}_{1.0} \mathrm{Hf}_{0.5} \mathrm{Ti}_{2} \mathrm{O}_{7}$ & $2 \% \mathrm{HfO}_{2}$ & $\mathrm{Hf}_{0.11} \mathrm{Ti}_{0.89} \mathrm{O}_{2}$ \\
\hline mws 980423 & $\mathrm{Ca}_{0.6} \mathrm{Gd}_{0.8} \mathrm{Hf}_{0.6} \mathrm{Ti}_{2} \mathrm{O}_{7}$ & & $\mathrm{Hf}_{0.11} \mathrm{Ti}_{0.89} \mathrm{O}_{2}$ \\
\hline mws 980424 & $\mathrm{Ca}_{0.7} \mathrm{Gd}_{0.6} \mathrm{Hf}_{0.7} \mathrm{Ti}_{2} \mathrm{O}_{7}$ & & $\mathrm{Hf}_{0.13} \mathrm{Ti}_{0.89} \mathrm{O}_{2}$ \\
\hline mws 980425 & $\mathrm{Ca}_{0.8} \mathrm{Gd}_{0.4} \mathrm{Hf}_{0.8} \mathrm{Ti}_{2} \mathrm{O}_{7}$ & & $\mathrm{Hf}_{0.14} \mathrm{Ti}_{0.86} \mathrm{O}_{2}$ \\
\hline
\end{tabular}




\section{Appendix A - X-ray Diffraction Results}

Samples were measured using a Siemens D500 Diffractometer, employing Co K-alpha radiation. 


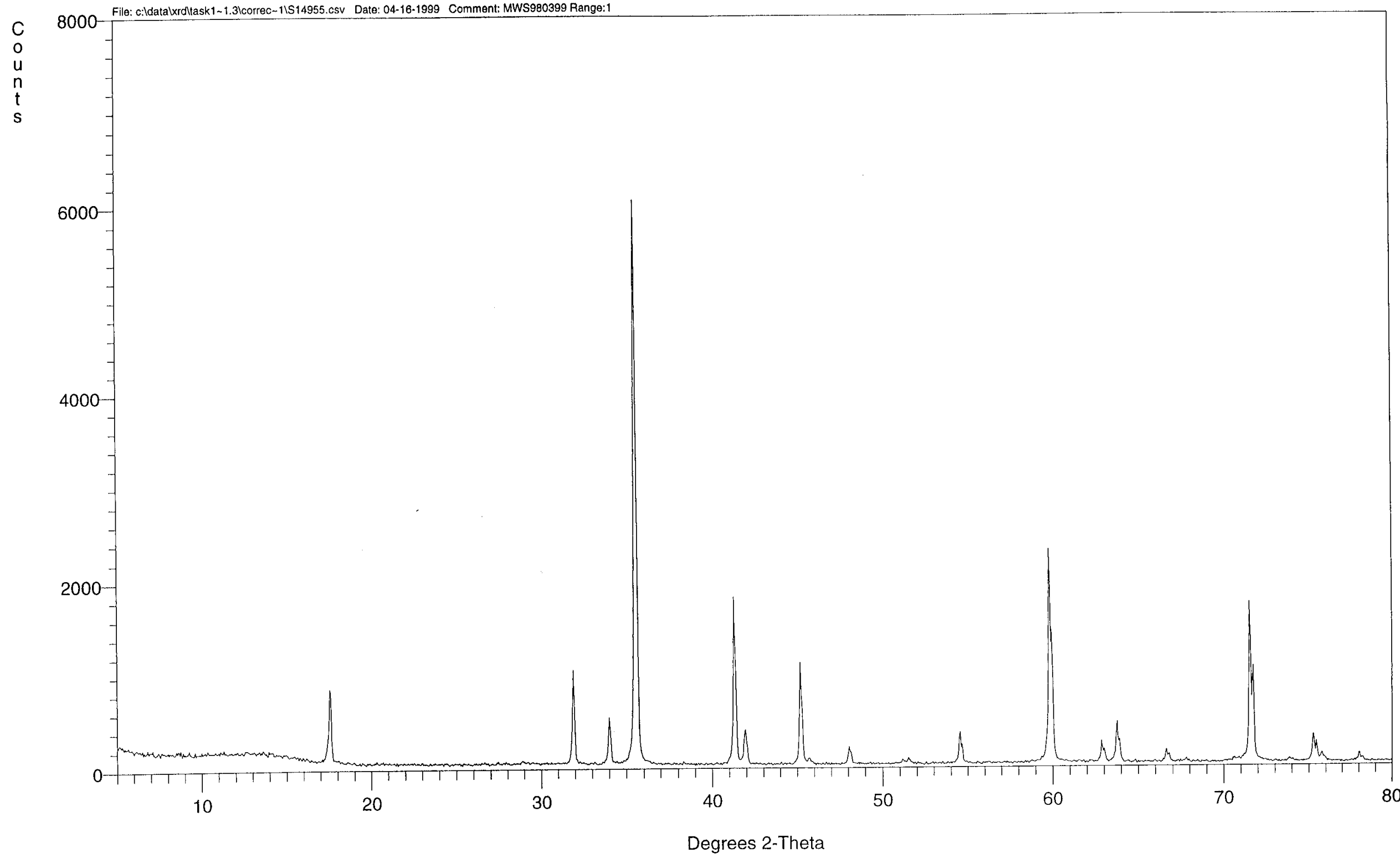




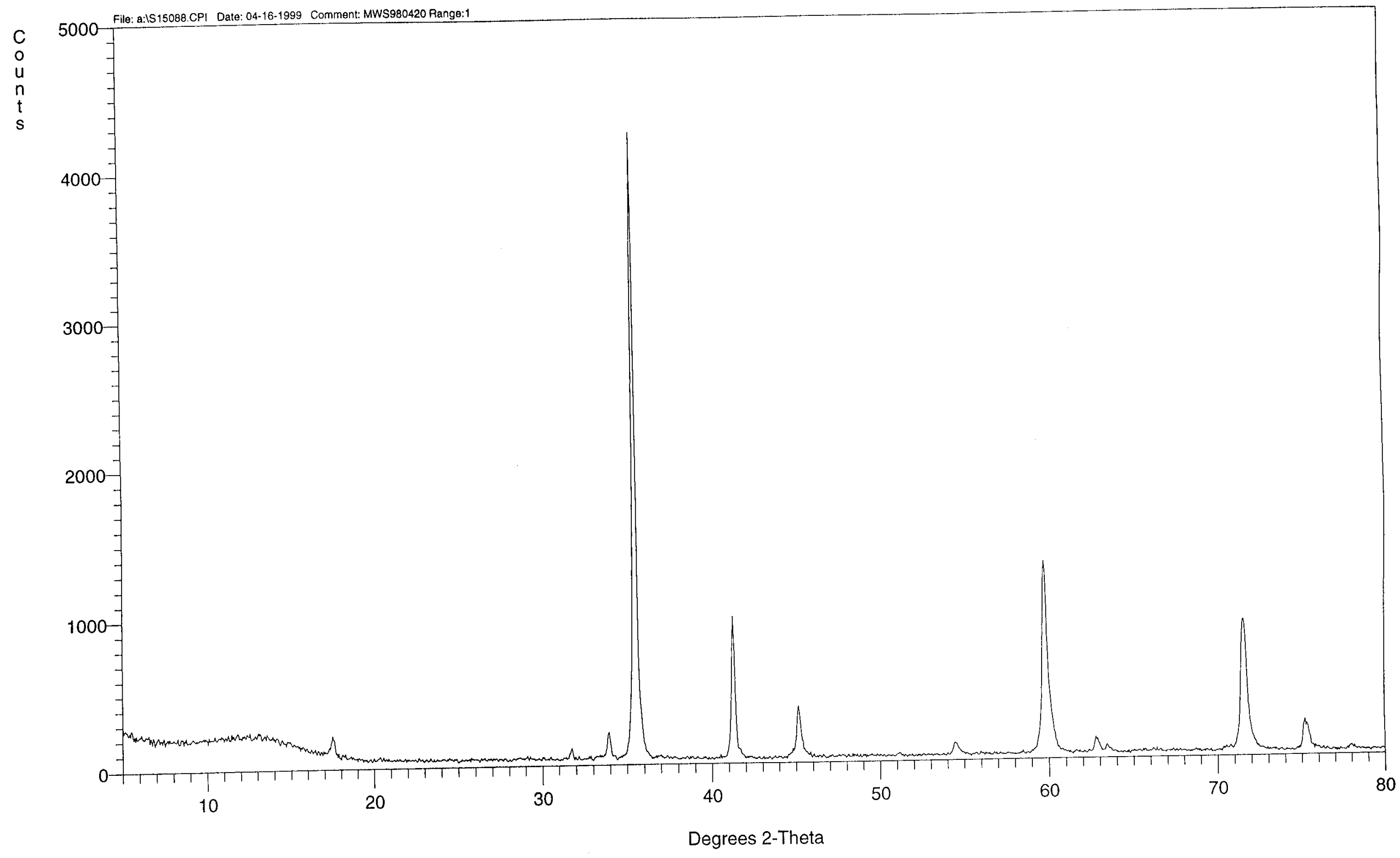




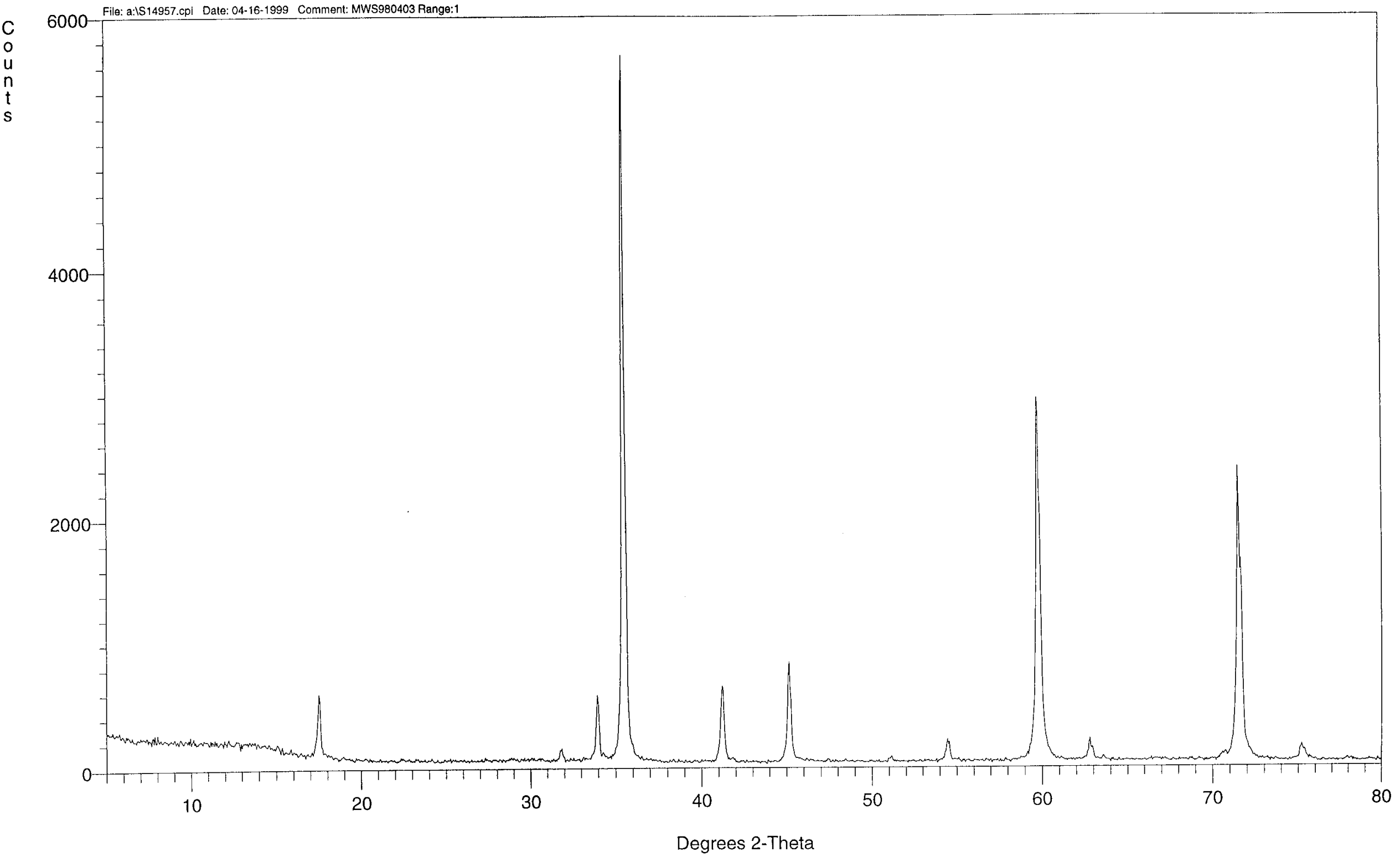




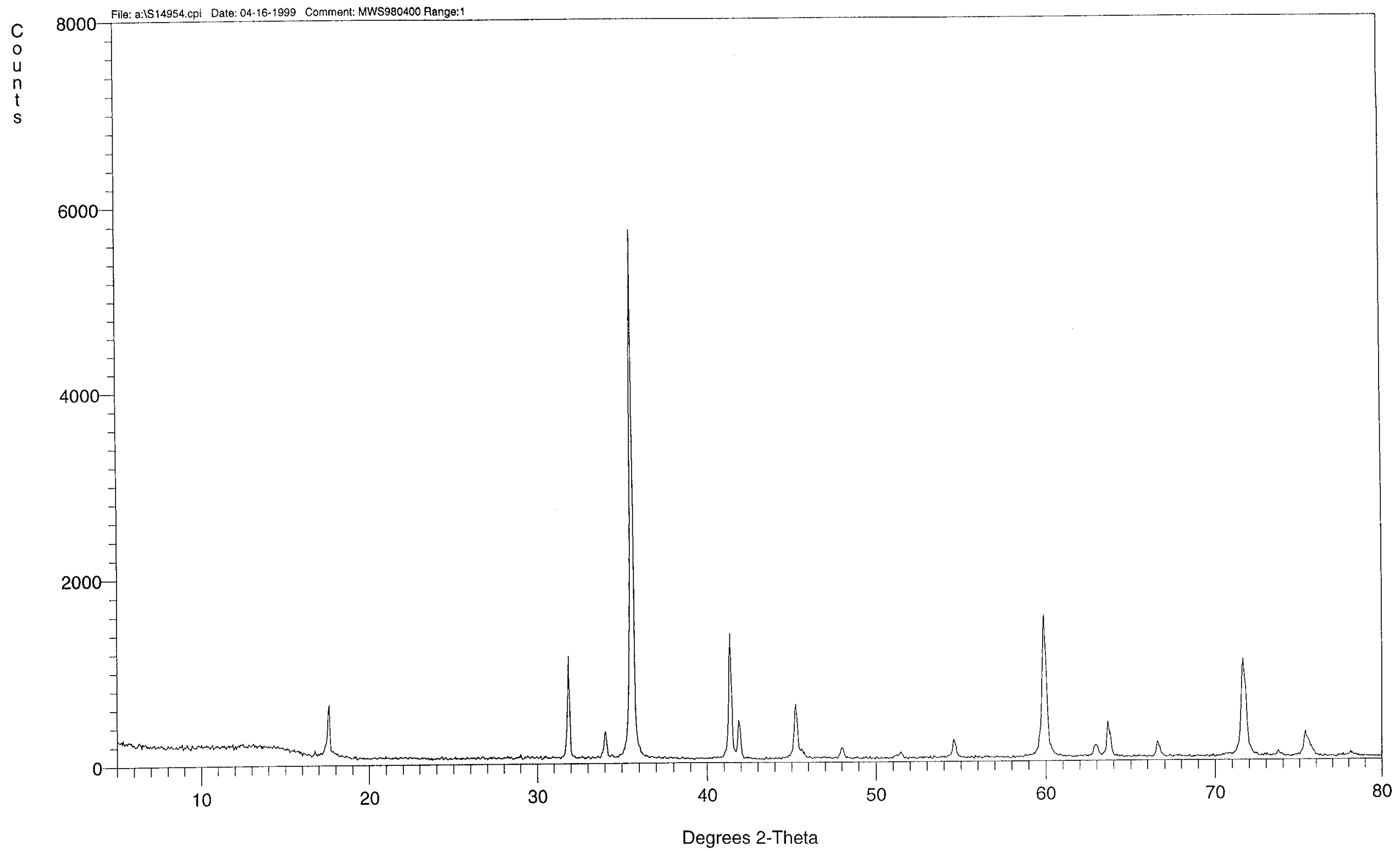




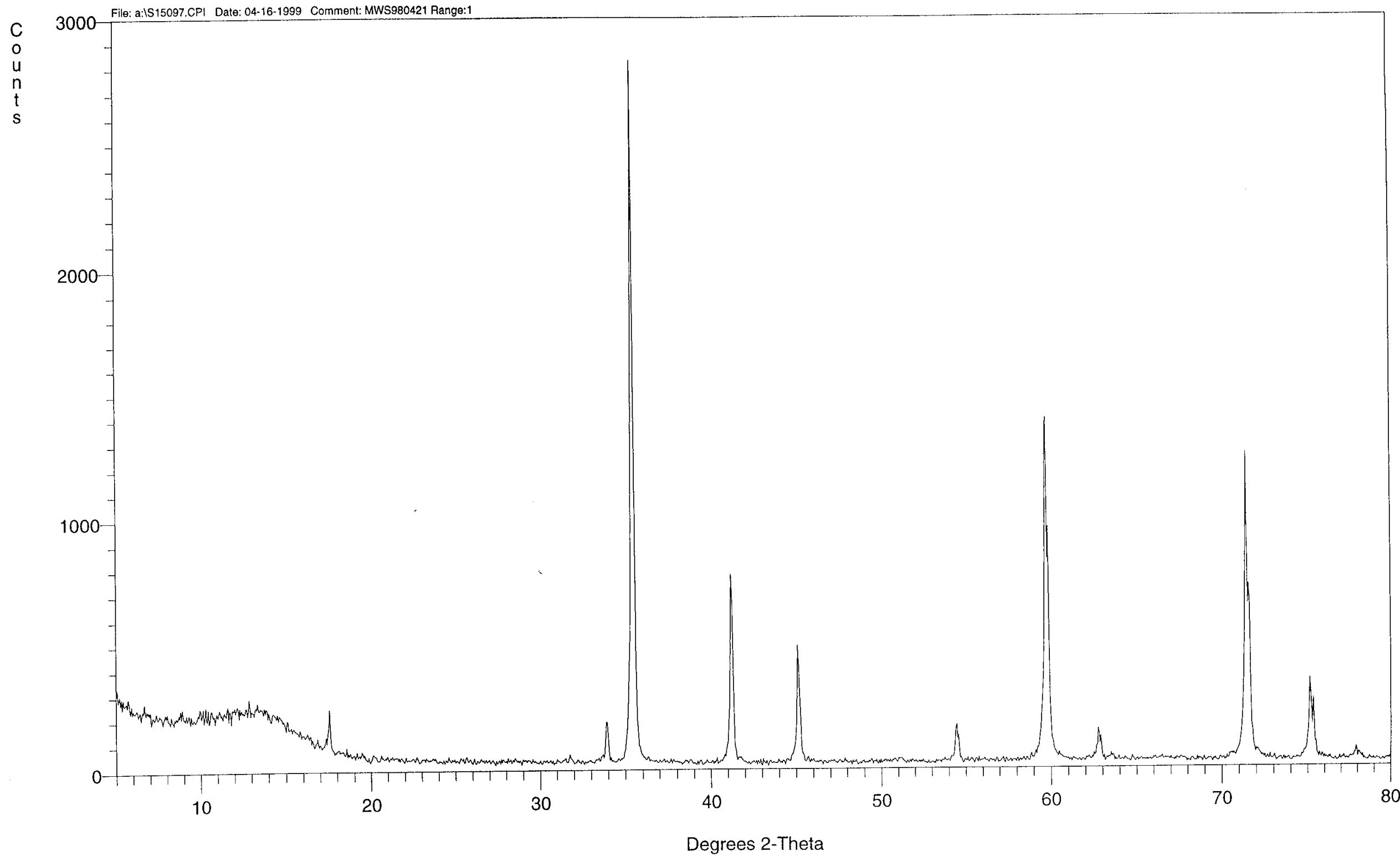




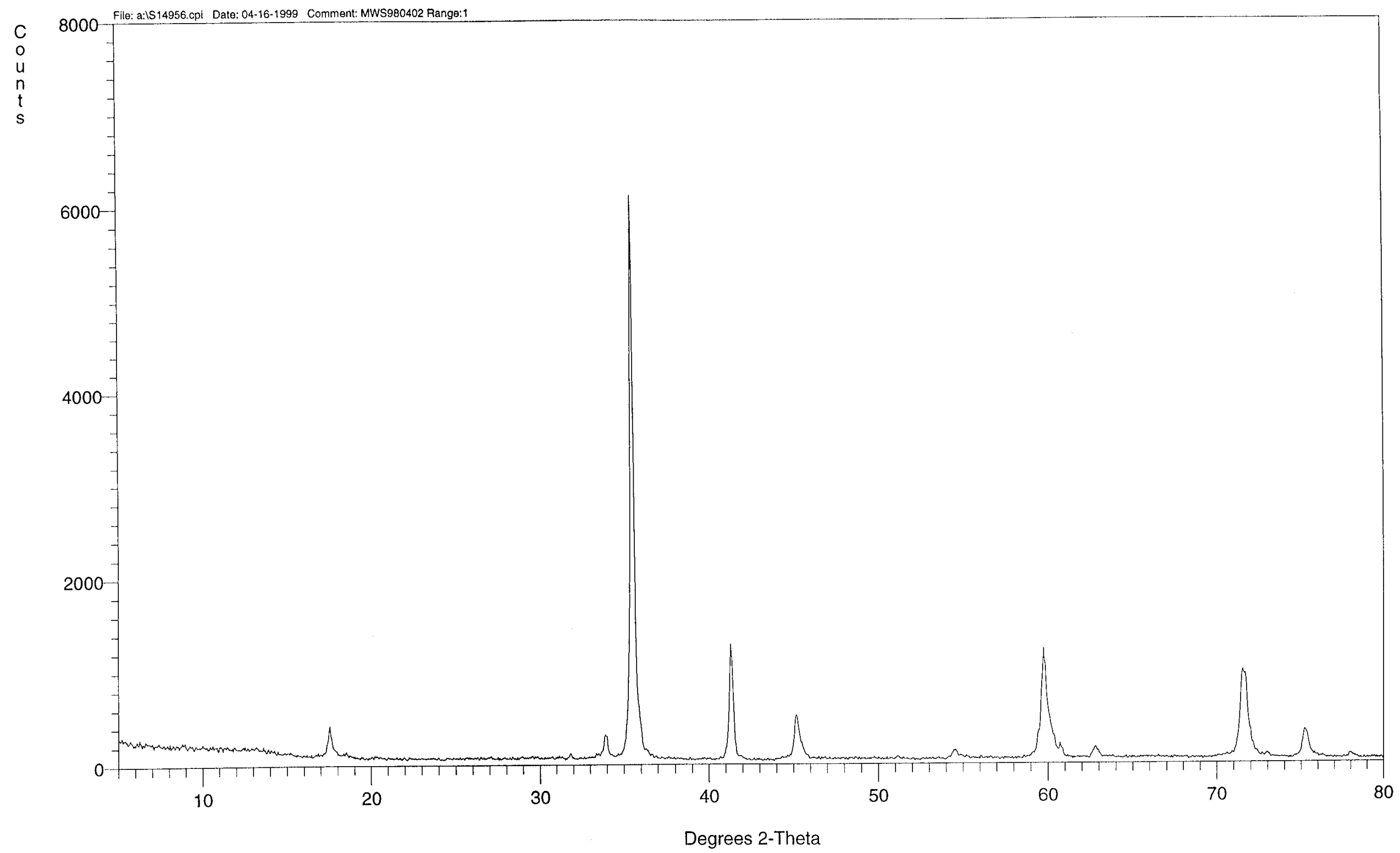




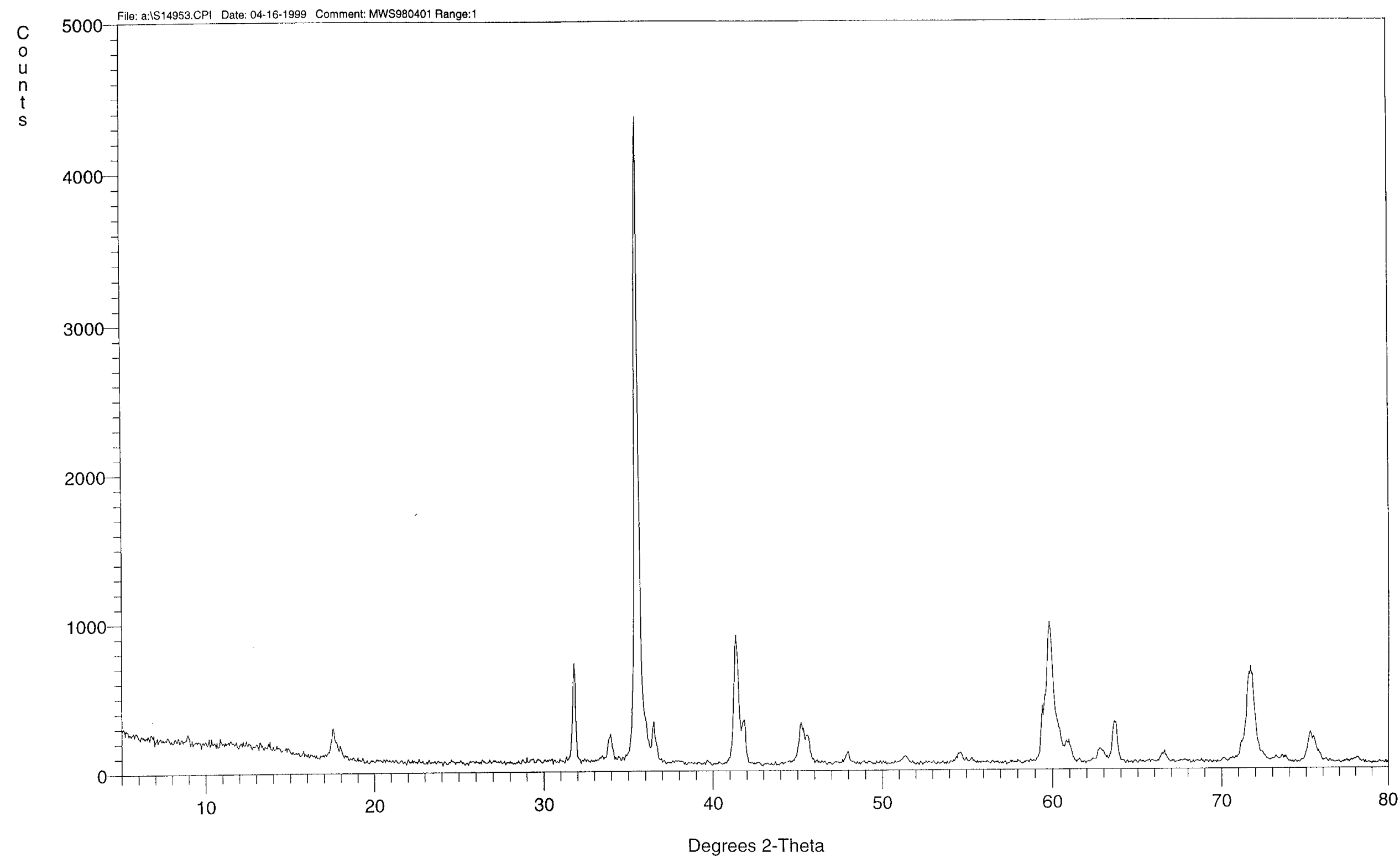




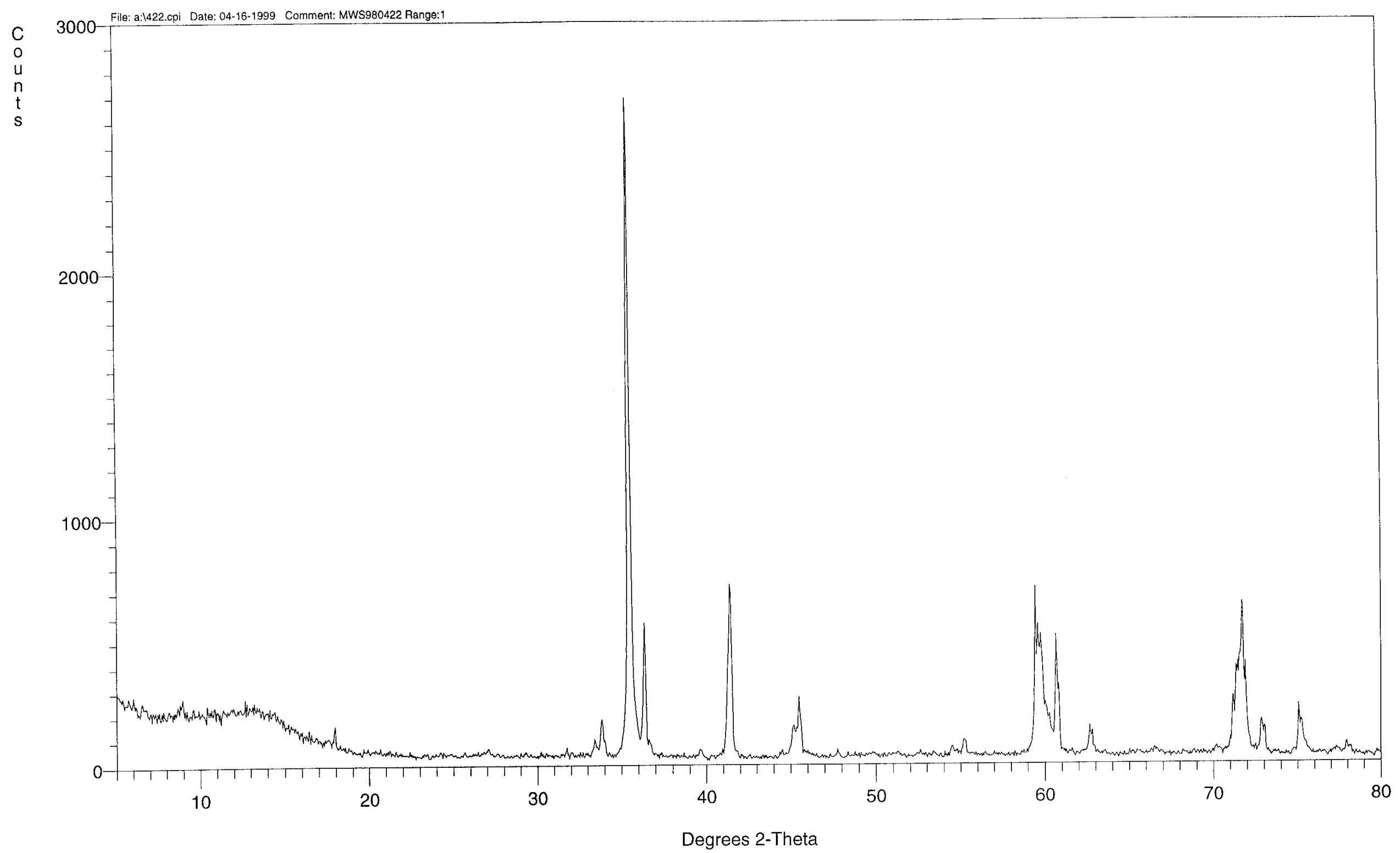




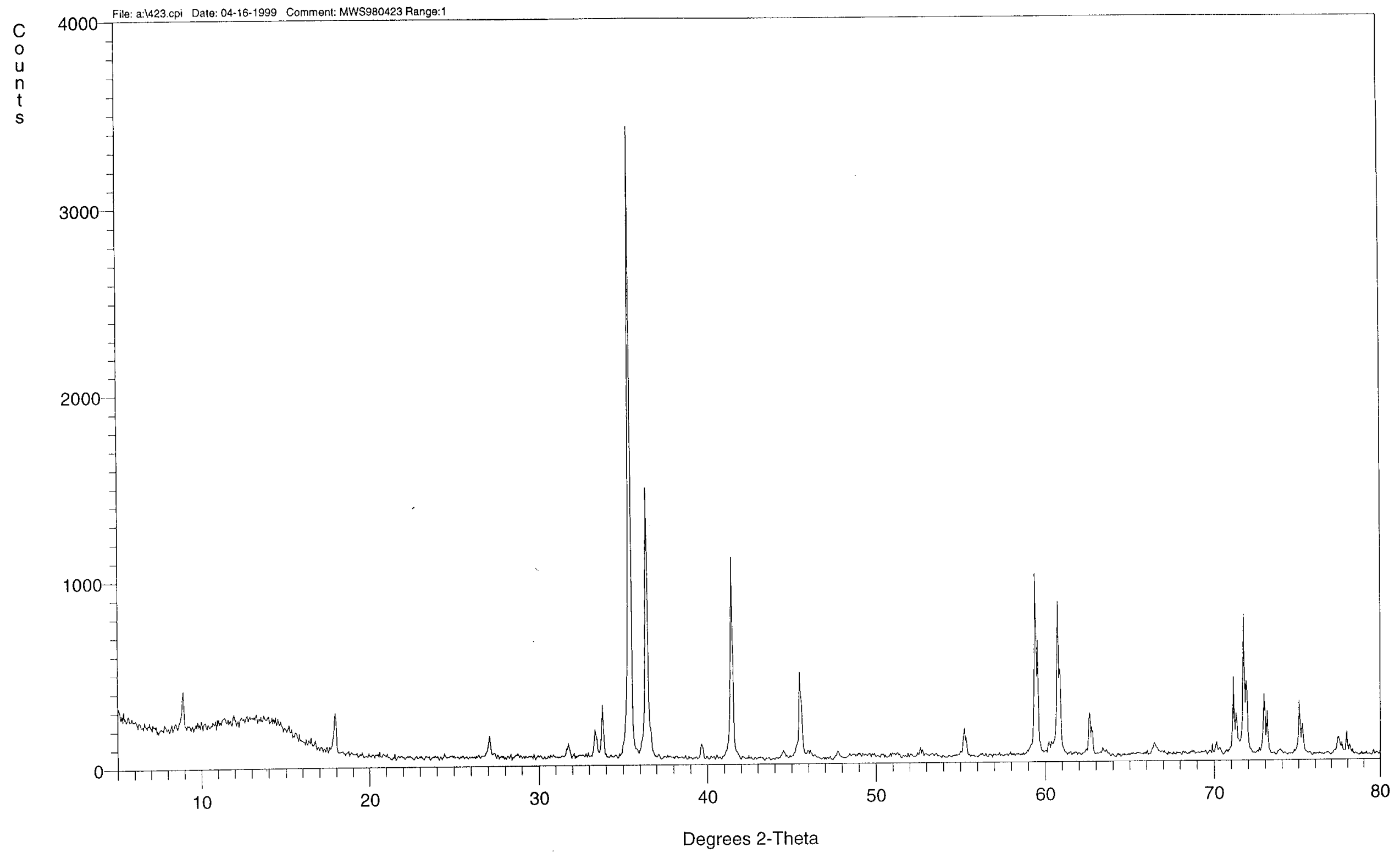




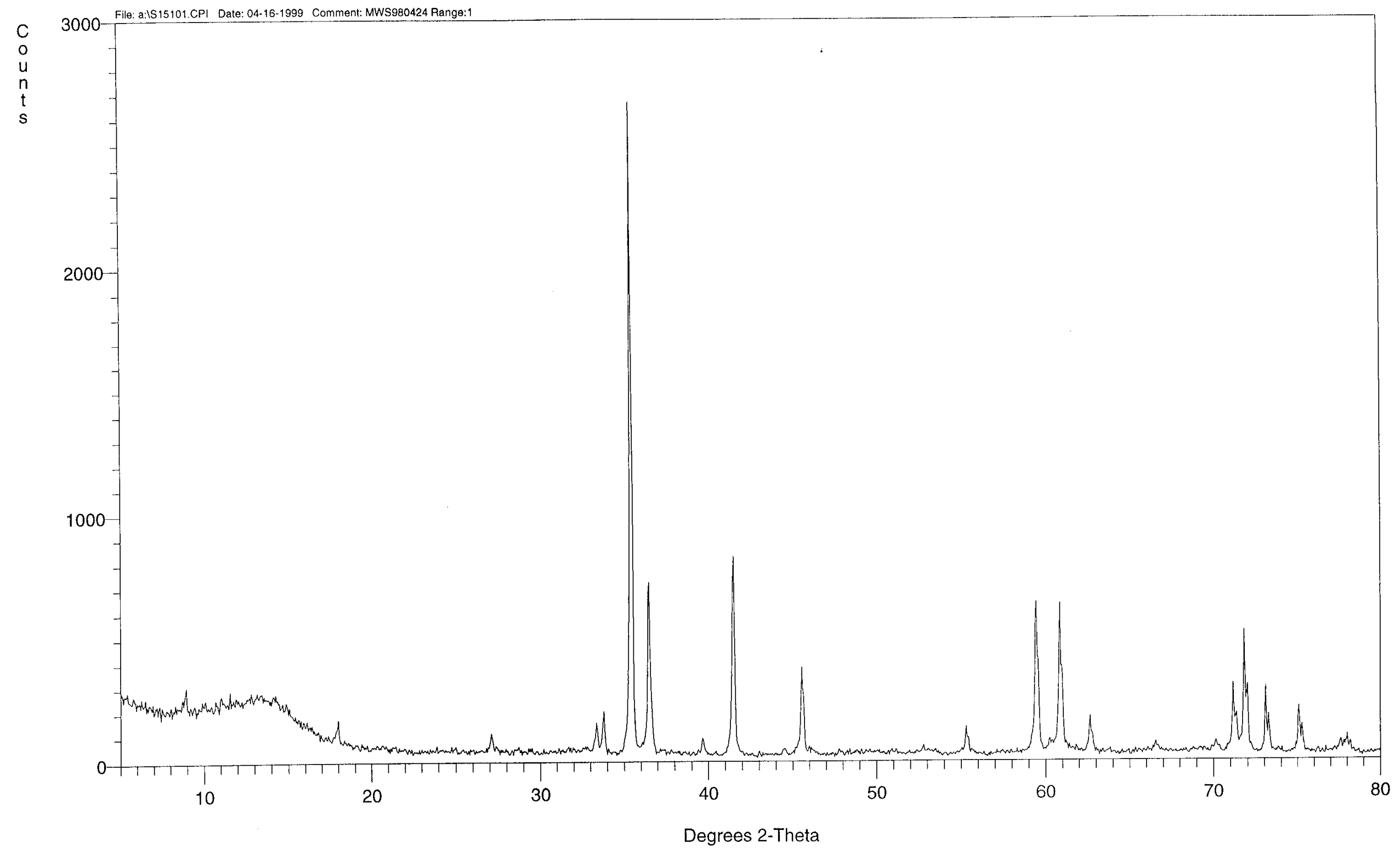




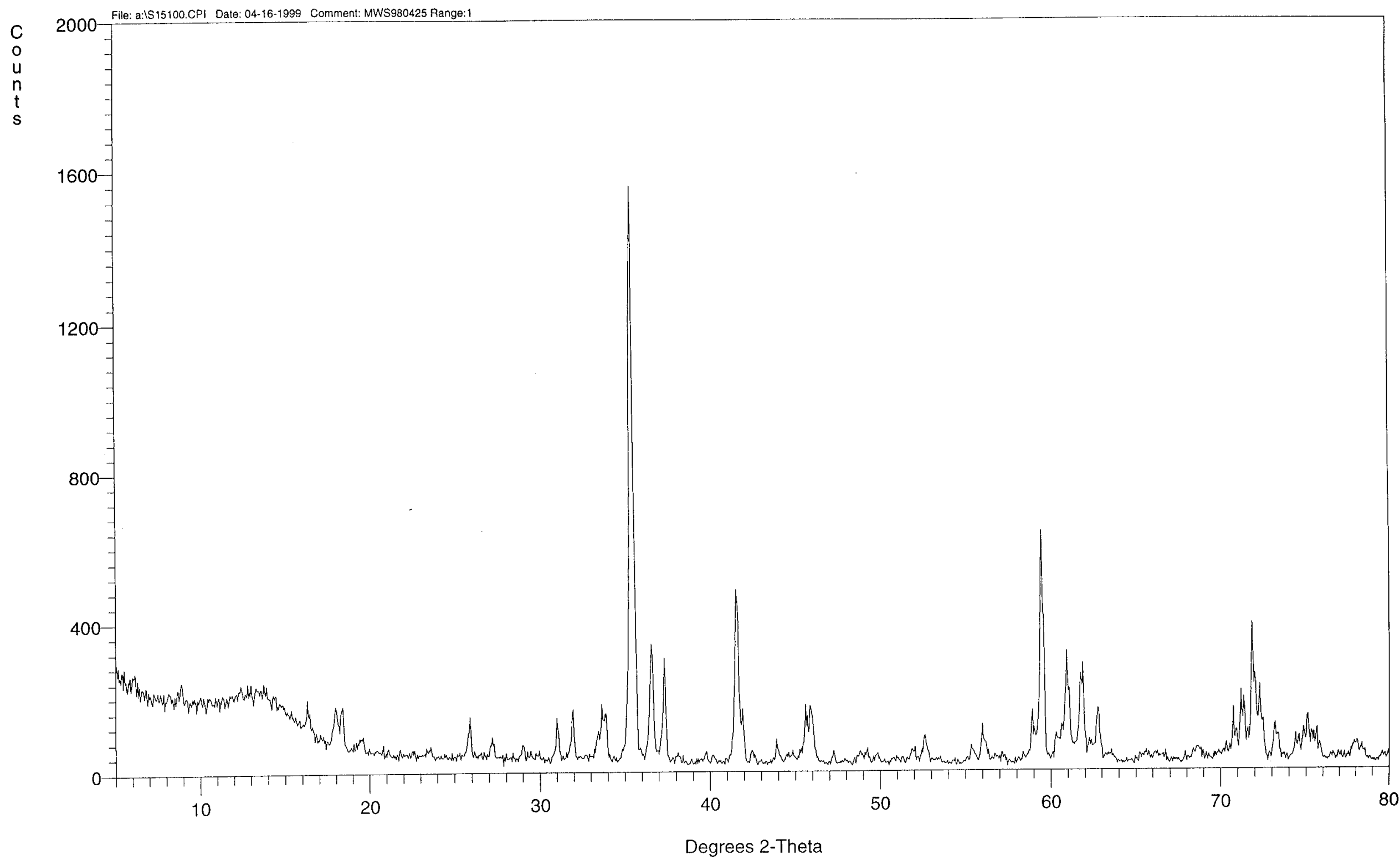




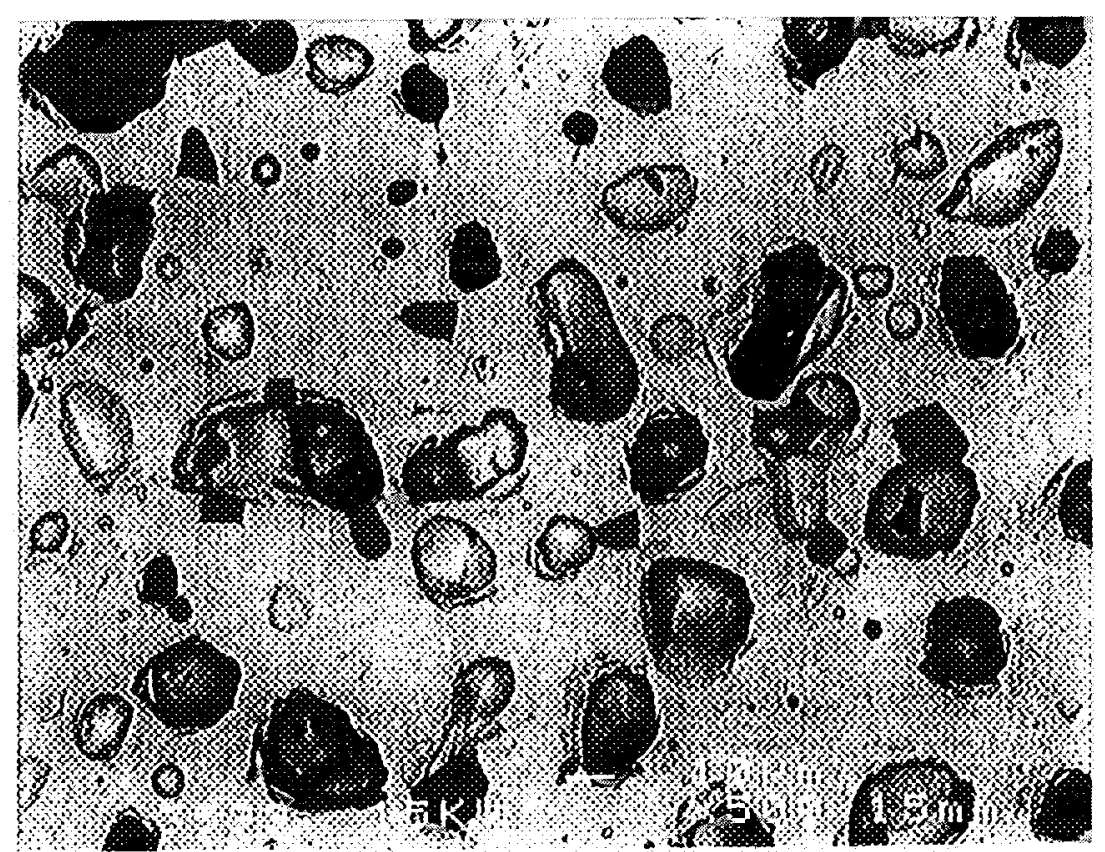

Figure 1: (a) Backscattered electron micrograph mws $980420\left(\mathrm{Ca}_{0.3} \mathrm{Gd}_{1.4} \mathrm{Hf}_{0.3} \mathrm{Ti}_{2} \mathrm{O}_{7}+2 \%\right.$ $\mathrm{HfO}_{2}$ ). The pellet consists of pyrochlore (matrix), with some rutile (dark-grey) and porosity.

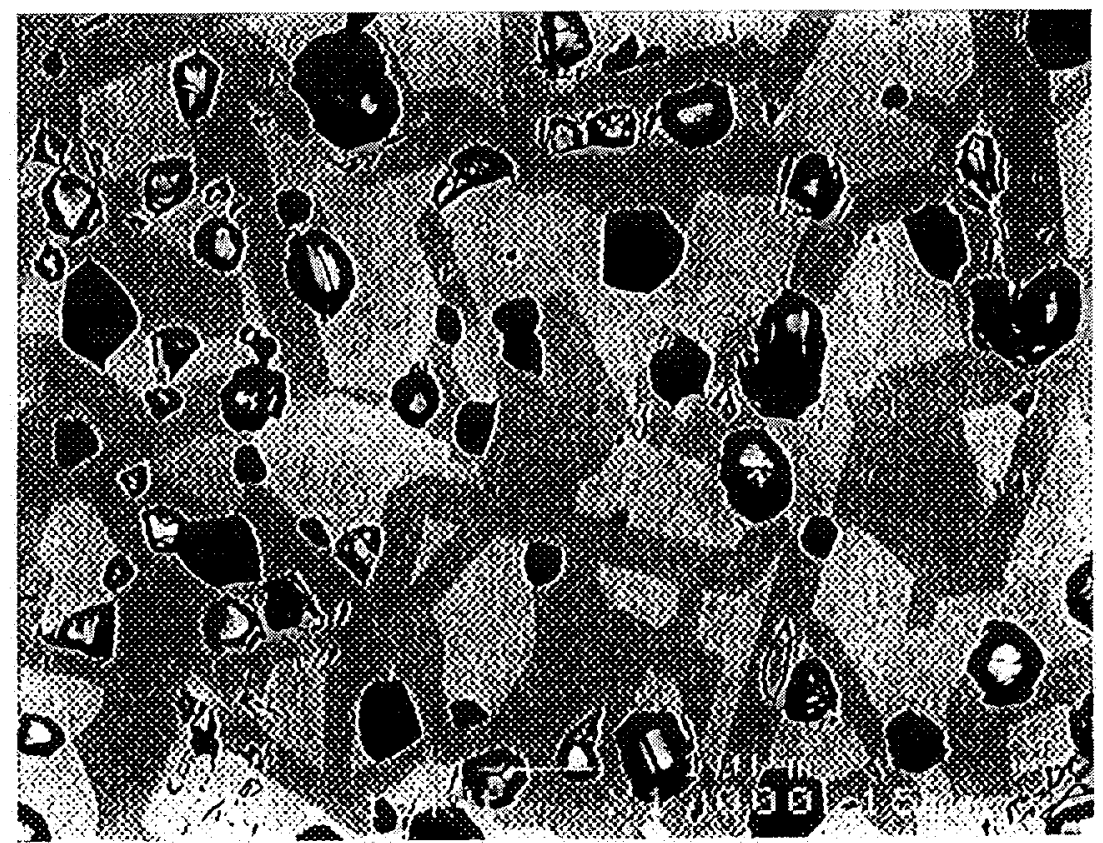

Figure 2: (a) Backscattered electron micrograph mws $980422\left(\mathrm{Ca}_{0.5} \mathrm{Gd}_{1.0} \mathrm{Hf}_{0.5} \mathrm{Ti}_{2} \mathrm{O}_{7}+2 \%\right.$ $\mathrm{HfO}_{2}$ ). The pellet consists of pyrochlore (light grey), $4 \mathrm{M}$ zirconolite (dark-grey) and porosity. 


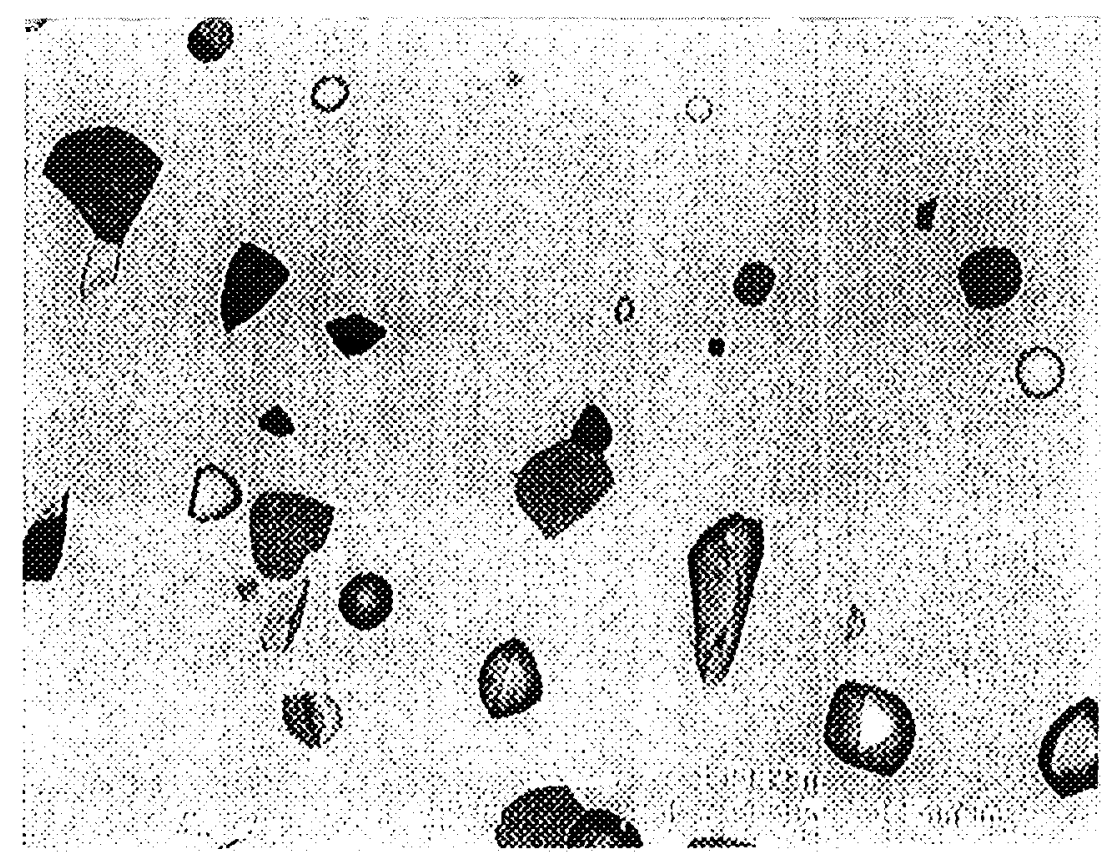

Figure 3: (a) Backscattered electron micrograph mws980423 $\left(\mathrm{Ca}_{0.6} \mathrm{Gd}_{0.8} \mathrm{Hf}_{0.6} \mathrm{Ti}_{2} \mathrm{O}_{7}\right)$. The pellet consists of a $4 \mathrm{M}$ zirconolite (matrix), with some rutile (dark-grey) and porosity.

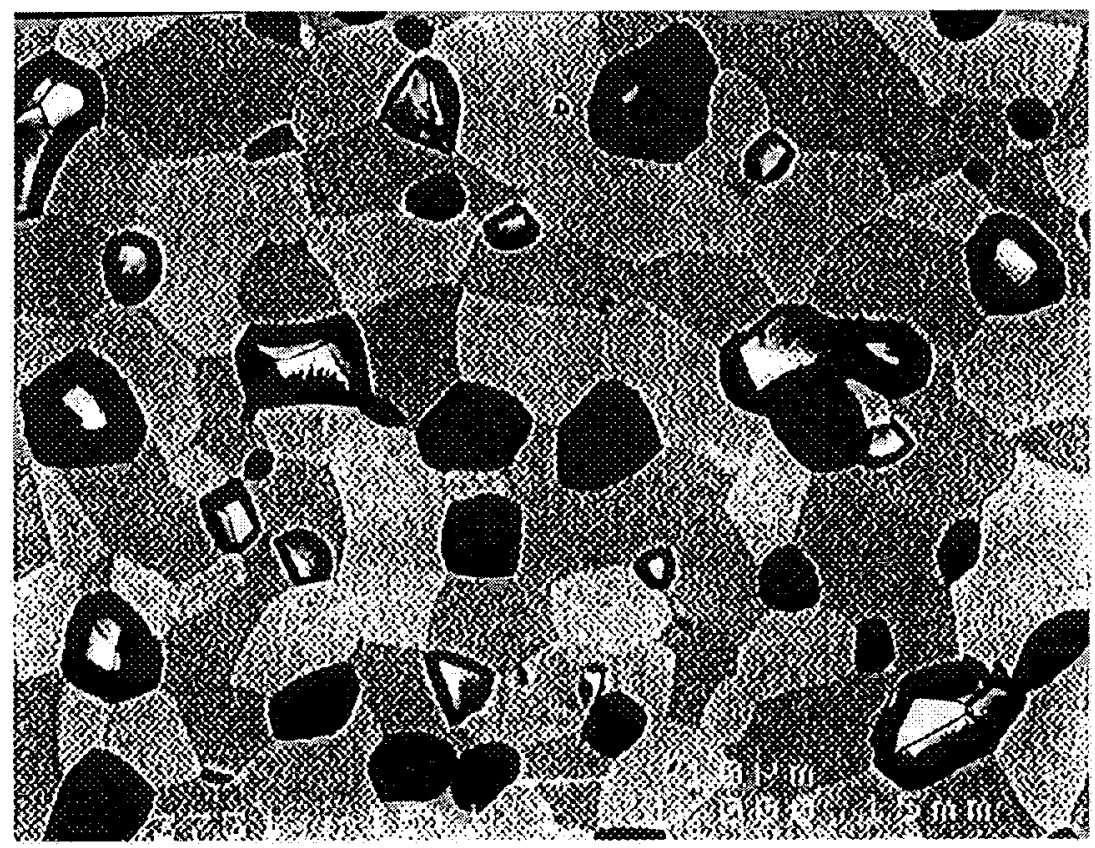

Figure 4: (a) Backscattered electron micrograph mws980425 $\left(\mathrm{Ca}_{0.8} \mathrm{Gd}_{0.4} \mathrm{Hf}_{0.8} \mathrm{Ti}_{2} \mathrm{O}_{7}\right)$. The pellet consists of $4 \mathrm{M}$ zirconolite (light grey), $2 \mathrm{M}$ zirconolite (dark-grey), rutile (darkgrey-black) and porosity. 